\title{
El Neurofeminismo frente a la investigación sobre la diferencia sexual
}

\author{
Neurofeminism on the sexual difference research
}

\author{
SONIA REVERTER-BAÑÓN*
}

\begin{abstract}
Resumen: En el presente trabajo se exponen las carencias más importantes en la investigación de la denominada "diferencia sexual en el cerebro". Desde la revisión y análisis crítico del grupo de científicas NeuroGenderings Network se propone una superación de los prejuicios que, de manera obvia o solapada, continúan manteniéndose en la investigación en relación a las diferencias sexuales en el cerebro. La tarea de "guerrilla epistemológica", como la he llamado, que este grupo feminista lleva a cabo con el fin de ir conformando un conocimiento rigurosamente científico se podrá ver con el ejemplo concreto de la propuesta que hacen a una revista neurocientífica. Palabras clave: Neurofeminismo, diferencia sexual, neurociencia de la diferencia sexual, neurosexismo.
\end{abstract}

\begin{abstract}
The aim of this paper is to analyze and expose the most important shortcomings in the investigation of the so called "sexual difference in the brain". Departing from the work of the academic group NeuroGenderings Network this paper will review and analyze critically its proposals to overcome the prejudices that, obviously or not, continue to be maintained in scientific research in relation to sexual differences in the brain. The task of "epistemological guerrilla", as we call it, that this feminist group carries out in order to conform a rigorous scientific knowledge can be seen with the concrete example of the proposal they have recently made to a neuroscientific journal.

Key-words: Neurofeminism, Sexual Difference, Neurosciences of Sexual Difference, Neurosexism.
\end{abstract}

\section{La diferencia sexual en el cerebro: ¿cien años de ignorancia?}

El pasado diciembre de 2015 la prestigiosa revista Proceedings of the National Academy of Sciences (PNAS) publicó un artículo que recoge los resultados de una amplia investigación sobre el cerebro humano. La autora principal de esta investigación, Daphna Joel, junto a su equipo tituló el artículo "Sex beyond the genitalia: The human brain mosaic". En él se afirma que no hay base científica para observar diferencias sexuales estructurales en el cerebro que puedan ser comparables a las diferencias que de forma

Recibido: 13/04/2017. Aceptado: 27/09/2017.

* Profesora de Filosofía de la Universitat Jaume I. Miembro del Instituto de Investigación Feminista de la misma universidad. El presente trabajo ha sido posible gracias a la ayuda del Proyecto de investigación de I+D+I del Ministerio de Economía y Competitividad FFI2016-76753-C2-2P. 
dimórfica y consistente observamos en los genitales. Es decir, que los cerebros humanos no pueden categorizarse en dos clases distintas. Su argumentación es la siguiente:

Here we show that, although there are sex/gender differences in brain and behavior, humans and human brains are comprised of unique "mosaics" of features, some more common in females compared with males, some more common in males compared with females, and some common in both females and males. Our results demonstrate that regardless of the cause of observed sex/gender differences in brain and behavior (nature or nurture), human brains cannot be categorized into two distinct classes: male brain/female brain (Joel et al, 2015: 1568) ${ }^{1}$.

Cien años antes de esta investigación, en 1915, el neurólogo Dr. Charles Dana² ofreció sus conocimientos profesionales en el entonces polémico tema del voto de la mujer en las páginas de opinión de The New York Times. El Dr. Dana advertía que las mujeres no son eficaces a la hora de evaluar iniciativas políticas, y por tanto el voto podría afectarles negativamente. Tanto que, advertía, tendrán un incremento del riesgo de demencia de un $25 \%$. La argumentación científica de este riesgo se asentaba en que la mitad superior de la médula espinal, que controla la pelvis y las extremidades, es menor en las mujeres. Como conclusión el Dr. Dana afirmaba que la participación política de las mujeres, es, por ello, “peligrosa para su salud" (en Fine, 2010: 131).

El contexto histórico del siglo XIX fue de gran auge en la investigación neuronal, y en concreto de investigación sobre las diferencias humanas, siendo especialmente relevante la que concierne a las diferencias sexuales. Fisiólogos como George Romanes han pasado a la historia como representantes no sólo de la diferencia sexual en el cerebro, sino por afirmar, de forma acientífica, cómo la diferencia de tamaño entre los cerebros masculino y femenino impediría por "muchos siglos" a las mujeres llegar a equipararse a los hombres en su potencia mental (Romanes, 1887: 666; en Wood, 2001: 172). El libro de Romanes, titulado ilustrativamente "Mental Differences Between Men and Women" comporta de sí un fallo cometido repetidamente, incluso hoy, a la hora de extraer consecuencias comportamentales (y mentales) no justificadas de diferencias cerebrales (en este caso el tamaño del cerebro). La jerarquía de los cerebros, como en los ejemplos de Romanes y de Dana, han supuesto en el pasado la elaboración de un discurso pseudocientífico que argumenta que existen diferencias innatas entre los sexos que reflejan diferencias en la capacidad mental. El viaje de estas afirmaciones pretendidamente científicas no ha ido, como podríamos esperar, de la experimentación a la conclusión, sino más bien al contrario: la supuesta "evidencia" empírica se utiliza para acomodar una jerarquía que se pretende "natural" (Reverter-Bañón, 2016).

1 Traducción propia: “Aquí se demuestra que, aunque existen diferencias entre el sexo y el género en el cerebro y el comportamiento, los seres humanos y los cerebros humanos están compuestos de "mosaicos" únicos de rasgos, algunos más comunes en las mujeres comparados con los hombres, algunos más comunes en los hombres comparados con las mujeres y algunos comunes en mujeres y hombres. Nuestros resultados demuestran que, independientemente de la causa de las diferencias observadas entre el sexo y el género en el cerebro y el comportamiento (naturaleza o crianza), los cerebros humanos no pueden clasificarse en dos clases distintas: cerebro masculino / cerebro femenino."

2 Este neurólogo es precisamente el que da nombre a la Dana Foundation, la cual organizó en 2002 el famoso congreso en San Francisco "Neuroethics: Mapping The Field", el cual da inicio a la neuroética como disciplina. 
Los cien años que separan las afirmaciones con las que hemos empezado este artículo, las del Dr. Dana y las de la neuropsicóloga Joel, son años de investigación tenaz, contundente y profusa sobre el cerebro humano. Es de esperar que el cúmulo de conocimiento científico adquirido en este tiempo ayude a formalizar las creencias sobre el cerebro de forma que vaya más allá de creencias culturales, meras opiniones o incluso prejuicios. La afirmación del Dr. Dana en 1915 no mostraba ningún estudio científico que apoyara sus palabras en The New York Times, más allá de la evidencia del tamaño de la médula espinal, la cual no comporta en ningún caso la legitimidad científica que necesitarían las conclusiones a las que llega el Dr. Dana. En contrapartida, la investigación de Joel y su equipo se basa en las resonancias magnéticas de 1.400 cerebros humanos, junto con los análisis de personalidad, actitudes, intereses y comportamientos de 5.500 personas más, para observar las diferencias sexuales estructurales en el cerebro "más allá de los genitales".

Podemos decir, de forma bastante convincente, que las palabras del Dr. Dana son fruto de sus creencias culturales, de su opinión y, probablemente, de sus prejuicios. Las afirmaciones de la neuropsicóloga Daphna Joel y su equipo son conclusiones de un proyecto científico de largo recorrido y utilizando la metodología requerida en este momento por los estándares científicos más exigentes. También es cierto que hay cien años de diferencia, y probablemente este dato nos pueda llevar a pensar que lo que se ha llegado a conocer en ese tiempo sobre el cerebro pueda llevar a conclusiones opuestas. Posiblemente esta sería la explicación en muchos temas que exigen investigación científica; es decir, que en cien años el conocimiento científico pueda haber llevado a conclusiones muy diferentes respecto a las que se mantenían (incluso de manera científica) hace un siglo. Sin embargo, a la dificultad del objeto de estudio, el cerebro humano, se suman otro tipo de dificultades, que son precisamente las que intentamos analizar en este texto; y que se relacionan, en general, con lo que podríamos llamar "pensamiento pseudocientífico", es decir, un pensamiento falso pero que conlleva creencias erróneamente consideradas científicas y basadas presumiblemente en el método científico o teniendo el estatus de verdad científica (Hansson, 2015).

La "mala ciencia" ha sido uno de los objetivos del cuerpo de conocimiento denominado "epistemologías feministas", la cual cuenta con una sólida tradición, tanto en la crítica a las epistemologías patriarcales como en las propuestas para superar la mala ciencia. Las diferentes teorías y modelos epistemológicos que elaboran tienen el objetivo de mejorar la ciencia, acercándose a una objetividad que pueda corregir los prejuicios sexistas y androcéntricos que ofrecen una visión parcial del mundo, orientada principalmente a descubrir (y a veces construir) verdades que sirven a intereses muy particulares, como son el control material y el mantenimiento de ciertas jerarquías sociales, económicas, políticas y culturales. Pensadoras como Sandra Harding, Helen Longino, Elizabeth Anderson, Miranda Fricker, Evelyn Fox Keller, Iris M. Young, Donna Haraway... son una buena muestra de elaboración de propuestas epistemológicas que buscan la verdad científica en sintonía con normas democráticas e igualitarias de funcionamiento.

En este contexto podemos entender la frase del historiador del sexo Thomas Laqueur en su clásico estudio de 1990 Making Sex: Body and Gender From the Greeks to Freud: "casi todo lo que se desea decir sobre el sexo ya ha sido reivindicado para el género" $(1994,33)$. Es decir, que el conocimiento científico sobre la diferencia sexual se encuentra 
profundamente sesgado por los mandatos de género, que no olvidemos que en el sistema patriarcal dominante comportan desigualdades entre hombres y mujeres precisamente basadas en las diferencias. Así, de forma acrítica sigue mantiéndose la diferencia como sustento de la desigualdad, algo profunda y profusamente denunciado por las teorías feministas del conocimiento. La comunidad científica parte así, en sintonia con el concepto feminista de "conocimiento situado", de una perspectiva de sujeto particular, de sujeto conocedor con una situación de género determinada, la de género varón. Esto es claramente cierto para gran parte de la ciencia relativa a la diferencia cerebral de los sexos producida en el siglo XIX y parte del siglo XX.

Como nos cuenta Cynthia Russett (1989) en su clásico texto Sexual Science: The Victorian Construction of Womanhood, podemos trazar cómo el interés del estudio científico de la humanidad a finales del siglo XIX y principios del XX vertebró el debate naturaleza/cultura en gran parte a través del estudio de las diferencias entre los sexos. En este debate se asume claramente que la naturaleza humana no es unitaria, sino diversa y separada. Tanto el color como el sexo, al ser las dos características físicas más evidentes, se convierten en objeto de estudio de esa diversidad, y pronto las disciplinas biológicas en crecimiento en la época de cambio hacia el siglo XX, como la fisiología, se centrarán cada vez más en el cerebro como el elemento que puede explicar los fundamentos de diferencias comportamentales, y rápidamente se pasa a utilizar estas para legitimar las posibles desigualdades. Textos clásicos como los de Ruth Bleier (1984), Ruth Hubbard (1990) y Ethel Tobach y Betty Rosoff (1994) lideraron el camino hacia la revisión crítica de las teorías biológicas sobre las mujeres. Si bien ese camino cuenta cada vez con métodos y teorías más conscientes y vigilantes de la calidad de la producción científica, no es menos cierto que el peso de lo ideológico, en este caso lo patriarcal, y de intereses espurios puede contaminar eventualmente la ciencia.

La producción de un conocimiento sexista es un claro ejemplo de construcción de no-conocimiento, como García Dauder y Pérez Sedeño (2017) afirman. El concepto de "epistemologías de la ignorancia" que Tuana acuñó $(2004,2006)$ visibiliza bien estas prácticas de no saber sobre el cuerpo de las mujeres. Esta autora cree necesario, no sólo entender y visibilizar las prácticas de conocimiento, sino también las prácticas responsables de la ignorancia, el no-conocimiento. En el caso de la diferencia sexual en los cerebros las manifestaciones de la ignorancia se relacionan con prácticas de poder, en el sentido de que la ignorancia, el no saber, se produce y mantiene desde posicionamientos de poder, como es la apelación a la misma autoridad científica. El discurso de la ignorancia produce así un no saber que se sitúa, desde el poder de la autoridad científica, como evidente, aun sin serlo. La fundamentación científica de tales discursos es nula o confusa, pero tiene la legitimidad y la fuerza de los poderes que la arropan. La resistencia a tal manifestación de ignorancia, la gestión de la ignorancia, como nos dice Spelman (2007), no sólo ha de desvelar la ignorancia y sus falsedades, sino proveer los conocimientos que han sido negados o suprimidos. Y esto será muy complicado si no se pueden romper los circuitos de producción y mantenimiento de conocimientos, los cuales, y para el caso que nos ocupa, están arduamente tramados con largos siglos de pensamiento patriarcal, con prácticas de opresión y exclusión, como hemos podido comprobar en el ejemplo del Dr. Dana. 


\section{La guerrilla epistemológica de NeuroGenderings Network (NGN)}

El surgimiento y desarrollo desde hace seis años de un movimiento de científicas cuyo objetivo es romper ese circuito de producción de ignorancia en el tema de las diferencias sexuales en el cerebro nos puede ayudar a desvelar cómo y en qué elementos concretos puede estar circulando, aún hoy, el no conocimiento neurocientífico de las diferencias sexuales. El denominado NeuroGenderings Network ${ }^{3}$ (NGN) se originó en un congreso en 2010 financiado por el Swedish Research Council, como parte del programa de excelencia GenNa: Nature/Culture and Transgressive Encounters, y por el Body/Embodiment Group ${ }^{4}$ del Center for Gender Research de la Universidad de Uppsala, organismo organizador de tal congreso. El congreso, con el título de NeuroGenderings: Critical Studies of the Sexed Brain, estaba motivado por el interés de ayudar a separar la investigación científica de la pseudociencia sobre la diferencia sexual del cerebro. A este congreso de 2010 le siguieron dos congresos más (Viena 2012, y Lausanne 2014). En ellos se ha ido juntando un grupo de científicas radicadas en diferentes universidades y centros de investigación de diversos países. Entre ellos encontramos el Instituto Pasteur de París, el Aston Brain Centre en Reino Unido, el Gender Research Office de la Universidad de Viena, Sagol School of Neuroscience de la Universidad de Tel Aviv, Barnard College o Brown University en Estados Unidos.

Esta red de trabajo es interdisciplinar, y aglutina disciplinas tales como neurociencias, humanidades, filosofía, ciencias sociales, estudios culturales, medicina, química, estudios de género y queer, teoría feminista y estudios de ciencia y tecnología. Su interés es investigar un amplio abanico de temas relacionados con el campo del sexo/género y el cerebro, evaluando el estado actual de la investigación neurocientífica, sus métodos, hallazgos y conclusiones, así como las representaciones e interpretaciones del cerebro. Su intención es iniciar un diálogo interdisciplinar para desarrollar propuestas neurocientíficas más detalladas y ricas que las que comúnmente se aportan en este campo. El objetivo es desplegar conceptos y debates más reflexivos para que puedan orientar mejor el diálogo entre las neurociencias y los intereses sociales y educativos (algo que esta red de trabajo denomina "neuropedagogía"). El punto de partida de tal investigación, análisis y crítica es la constatación que muchas de las conclusiones neurocientíficas sobre las diferencias sexuales del cerebro no sólo no están fundamentadas científicamente, sino que obedecen a planteamientos (conscientes o no) e intereses (voluntarios o no) neurosexistas. El término "neurosexismo" fue acuñado en 2008 por primera vez por la psicóloga Cordelia Fine, miembro actualmente del grupo NeuroGenderings Network. El término refiere a las posturas sexistas, acríticas y acientíficas que pretenden demostrar que el cerebro de mujeres y hombres es diferente simplemente por ser mujeres y hombres. El grupo NGN pronto acuñó el neologismo de "neurofeminismo" como respuesta crítica. Lo que pretenden es hacer una revisión crítica de las teorías científicas para depurar lo ideológico patriarcal que pueda haber en las teorías y experimentación sobre la diferencia sexual del cerebro. A través de la lista de distribución mantienen lo que desde aquí llamamos una "guerrilla epistemológica" que se mantiene vigilante ante cualquier publicación que pueda quebrar los principios de la buena ciencia en el área concreta de las

3 https://neurogenderings.wordpress.com/

4 http://www.genna.gender.uu.se/themes/bodyembodiment/ 
neurociencias. El punto de partida de este grupo es la constatación de que la investigación sobre las diferencias entre los cerebros masculino y femenino se basa en resultados falsos, de mala calidad, malas metodologías, supuestos no probados y conclusiones prematuras (lo que antes hemos denominado con Tuana, epistemología de la ignorancia).

\subsection{Sexo/Género, Biología/Cultura y plasticidad cerebral}

Con vistas a poder mejorar el conocimiento científico sobre las diferencias sexuales en el cerebro y en la cognición humana el NeuroGenderings Network mantiene una lista de distribución gestionada desde la Universidad de Viena. El equipo de personas integradas en esta lista participa desde 2012 en una conversación permanente en la que se revisan tanto los artículos y publicaciones científicas como divulgativas en relación al tema de la diferencia sexual en los cerebros humanos. Una de las preocupaciones constantes generadas en la conversación que en esta lista se mantiene es la usual y casi permanente confusión sobre los conceptos de sexo y género en las publicaciones científicas ${ }^{5}$. Se trata de una confusión de gran trascendencia, pues los conceptos de sexo y género van a estructurar el marco de investigación sobre las diferencias y/o similitudes sexuales. Separarlos como analíticamente diferentes o unirlos, entendiéndolos en co-implicación superando así la dicotomía naturaleza/cultura, son dos posicionamientos sobre los que las mismas teorías feministas están debatiendo desde hace décadas (Nicholson, 1994; Scott, 1997). Ahora bien, confundirlos, como algunas investigaciones científicas sobre el cerebro hacen, produce un efecto distorsionante que anula cualquier posible conclusión científica. Por ello, aclarar los conceptos de sexo y género es el primer paso para acabar con los persistentes equívocos en la investigación sobre el cerebro humano.

La confusión entre sexo y género llega a ser tan grande que las diferencias entre mujeres y hombres (independientemente de si son neuronales o comportamentales) son a veces llamadas "diferencias de sexo" y a veces "diferencias de género". Como ejemplo, una de las miembros del NGN, Kaiser $(2012,131)$, incide en la constante confusión terminológica en los casos de importantes publicaciones neurocientíficas como Cerebral Cortex o Nature Neuroscience. Los estudios de Gur et al., (2002, en Kaiser 2012), publicados en la primera, nos hablan de diferencias sexuales en el volumen cerebral frontal, y los estudios de Lüders, Narr, \&Thompson (2004, en Kaiser 2012), publicados en la segunda revista, establecen las diferencias de género en la complejidad cortical. Como la misma Kaiser nos advierte, esta indecisión terminológica puede bien reflejar una incerteza más profunda acerca de cómo observar y explicar el origen de las diferencias entre hombres y mujeres. Como Schmitz (2012) ha observado ello implica que hay inseguridad y/o desacuerdo respecto a si las diferencias comportamentales entre hombres y mujeres son atribuibles al sexo del cerebro, o al contrario, si esas diferencias en el cerebro son explicables por las diferencias en el comportamiento.

5 También preocupa esta confusión en las publicaciones divulgativas, en las que además es más llamativa por el alcance de público que tienen tales publicaciones. Aunque el grupo NGN se ocupa principalmente de evaluar y ayudar a corregir tesis y experimentos en las mismas revistas científicas que los publican, no es inusual que de forma esporádica emitan alguna nota de prensa o escriban algún artículo en medios divulgativos. Como ejemplo podemos poner el artículo de opinión aparecido recientemente en el periódico The Guardian (6 de abril de 2017), con el ilustrativo título de "We've been labelled 'anti-sex difference' for demanding greater scientific rigour" (firmado por Cordelia Fine y Rebecca Jordan-Young). 
La cuestión es que, si bien es cierto que las diferencias psicológicas y psico-sociales entre los sexos pueden llegar a ser grandes y están bastante bien descritas, las diferencias en el cerebro no son tan evidentes. A la vez, tampoco es obvio ni científicamente reconocido el nexo entre las primeras y las últimas. Es decir, no está científicamente probado que las diferencias psico-sociales sean producto de las diferencias cerebrales entre sexos. Como ya hemos mencionado, la confusión sexo y género nos remite al debate sobre naturaleza y cultura. Resulta curioso, sin embargo que el marco de la confusión de estos conceptos sea uno de rígida separación, incluso podríamos decir dicotomía, entre naturaleza y cultura. Se separa naturaleza y cultura pero se confunden, e incluso se llegan a utilizar de forma intercambiable, sexo y género. Ello remite a un marco ideológico, que podemos entender que es el patriarcado, que asume y parte de la idea que la diferencia sexual es una diferencia de género; que las diferencias sexuales en el cerebro (aceptando que las haya), explican las diferencias de género. Parece así tratarse, como señalan Severin y Wyer (2000), de un "impulso teórico" que lleva a entender la biología humana como un "gancho" del que pende la cultura. Como ha sido constantemente señalado por las teorías feministas esto es la naturalización de las diferencias, que sirven para, indirectamente, naturalizar a su vez las desigualdades.

Es importante indicar que la denuncia desde posicionamientos feministas de la confusión constante entre sexo y género no pretende deshacer el enredo separando de forma dicotómica tales conceptos, sino precisamente encontrando un marco explicativo que sin confundirlos nos proponga articularlos en relación constante y fluida. Así, la reconocida bióloga FaustoSterling (2000), miembro del NGN, propone entender la relación sexo/género desde la teoría de sistemas dinámicos, ya que ofrece un marco más apropiado y productivo que el que proporciona la vieja dicotomía naturaleza/cultura. Según su propuesta, y tal como algunas teóricas del feminismo también nos proponen (Butler, 1990; Spivak, 1993; Grosz, 1994) ambos, sexo y género, son parcialmente constructos sociales, aunque toman forma en el cuerpo, y por ello son simultáneamente biológicos. La teoría de los sistemas dinámicos nos permite unir lo social y lo biológico, y entender así cómo la diferencia cultural se convierte en diferencia biológica. Fox Keller (2010) nos habla de la necesidad de superar la dicotomía biología y cultura para encontrar un lenguaje que refleje mejor la ciencia contemporánea y nos ayude a salir de "la marisma en la que hemos estado atascados tanto tiempo" (Fox Keller, 2010, 13).

De alguna manera esta propuesta está en sintonía con la idea de biocultura como el concepto que mejor recoge la realidad de lo que es la especie humana. La propuesta del concepto de biocultura ve la pregunta por la relación de la naturaleza y la cultura, no como el problema, sino como la solución (Ridley, 2003). Lejos de querer delimitar el campo de acción de cada uno de estos dos conceptos propone entender que en el ser humano no se pueden separar. En esta línea podemos situar el creciente interés por el papel de la plasticidad neuronal, concepto que conllevaría un entendimiento cada vez más consolidado de la importancia y peso de los contextos culturales y experienciales en los mecanismos neuronales y en las actividades genéticas (Li, 2003). Ello ya es propuesto por algunas como un camino para una colaboración más fructífera entre las diversas disciplinas que estudian el ser humano (Davis \& Morris, 2007), y en especial entre las neurociencias y las ciencias humanas y sociales (Gottlieb, 2000, 2002; Greenfield, 2000). 
Es desde este acercamiento de revisión crítica que podemos entender la tarea de observación que realiza el grupo NGN. A modo de ejemplo relataremos el caso reciente en el que cinco de las líderes del grupo han escrito una nota a la revista Journal of Neuroscience Research, una revista importante en el campo de la investigación neurocientífica.

\section{El sexo como variable biológica: el caso de Journal of Neuroscience Research}

En el año 2014 el National Institute of Health (NIH) de Estados Unidos anunció que implementaría un nuevo plan de política de financiación de la investigación ${ }^{6}$ (Clayton \& Collins, 2014). Este consistió en exigir (a partir de enero de 2016) la inclusión del sexo como variable biológica (el conocido como SABV) en las investigaciones preclínicas animales y con humanos, con el objetivo de corregir posibles disparidades en la salud de los dos sexos. La idea que respalda esta decisión es que hay muchos desórdenes de salud que afectan de forma diferente a hombres y mujeres, y en consonancia el tratamiento médico y farmacéutico requerirá ser diferente.

Esta nueva política motivó, principalmente en Estados Unidos, a partir de 2014, un debate público, no sólo entre los y las profesionales e investigadores, sino también en el público general. El debate se ha mantenido en conferencias, congresos y workshops, así como en revistas científicas, y también en medios de comunicación masiva, como periódicos y revistas de divulgación. El impacto que ha tenido esta nueva política ha llevado a muchas revistas científicas a trasladar esta exigencia a las investigaciones que publican.

Así es el caso de la revista Journal of Neuroscience Research, la cual en su editorial del número de enero de 2017 anuncia un cambio en su política editorial en correlación con la exigencia del NIH. Este consistirá en "requerir a todos los autores asegurarse de la consideración apropiada del sexo como una variable biológica" (Prager, 2017, 1). El número en el que se anuncia este cambio trata de manera monográfica, además, el tema de la diferencia sexual en el cerebro ${ }^{7}$. El monográfico tiene el prometedor título de An Issue Whose Time Has Come: Sex/Gender Influences on Nervous System Function. En este número, por tanto, se anuncia una nueva política editorial y se presentan artículos que investigan las diferencias sexuales en el cerebro.

El equipo de NeuroGenderings Network (NGN) entiende que la nota editorial de la revista Journal of Neuroscience Research necesita ser matizada con criterios específicos sobre cómo asegurar esa "consideración apropiada del sexo como una variable biológica". Y ello es lo que hacen varias neurocientíficas del NGN en una reciente carta al editor publicada en un número posterior de la misma revista (22 de febrero 2017). Las investigadoras Rippon,

6 En la Unión Europea contamos con el programa de la Comisión Europea “Horizon 2020”. Uno de sus objetivos es integrar la dimensión de género en la investigación e innovación, tanto en su programación y planificación como en contenidos (Véase "Promoting Gender Equality in Research and Innovation", en https://ec.europa.eu/ programmes/horizon2020).

7 Tengamos en cuenta que en principio se trata de dos cuestiones diferentes: una es la exigencia del NIH y de la revista en cuestión de considerar el sexo como variable biológica en todo tipo de experimentos y pruebas preclínicas; la otra cuestión es la investigación de las diferencias sexuales. En este número de esta revista coinciden las dos: ofrece un monográfico sobre diferencias sexuales (en concreto en el cerebro y en la cognición), y se publica la nota editorial haciéndose eco de la nueva política del NIH. 
Jourdan-Young, Kaiser, Joel y Fine (2017) ofrecen en esa carta comentarios constructivos a este respecto. En especial sus advertencias van dirigidas a la investigación neurocientífica con humanos, ya que es en los seres humanos donde las potenciales influencias de un conjunto amplio de variaciones deberían ser consideradas y valoradas.

La primera apreciación que hacen es aclarar que si bien hay dos fuentes de influencia biológica clara, como son los genes XX versus $\mathrm{XY}$, y las hormonas gonadales; hay, a su vez, muchos factores adicionales que pueden influir en las diferencias entre sujetos femeninos y masculinos, como Joel y McCarthy (2017) o Ainsworth (2015) también señalan. Diferencias en los genitales, en el tamaño global o en la masa muscular, por ejemplo, pueden tener influencia en el comportamiento. Así, el peso global de una persona puede ser más importante que el sexo a la hora de calcular la dosis de un fármaco. Los atributos físicos impactan, además, en la forma como interactuamos a nivel individual. Y ello además, tiene correlación con el componente de género, con las expectativas sociales según el género y con los comportamientos en relación a las normas diferenciadas por sexo en las sociedades (Joel y MacCarthy, 2016: 2).

Como Eliot y Richardson (2016) han alertado, el nuevo requisito del NIH tiene el objetivo de visibilizar las diferencias de salud en relación al sexo, pero tal mandato puede no ser siempre la solución, otras cautelas habrán de incluirse. Como estas autoras señalan las disparidades en la salud humana en referencia al sexo tienen un fuerte componente sociocultural íntimamente vinculado al sexo biológico, que puede no tenerse en cuenta si no se estudia la variable de género, o nos remitimos a la base del modelo animal. Según aclaran Eliot y Richardson (2016) la exigencia de introducir la variable de sexo en la investigación animal parte de la presunción de que el cerebro humano y las diferencias comportamentales tienen un origen eminentemente biológico, despreciando así la importancia que los aspectos sociales, psicológicos y culturales puedan tener en las diferencias neuronales entre hombres y mujeres. Esta asunción, sin embargo, es contraria a algunas de las conclusiones relacionadas con la plasticidad cerebral y la epigénesis, las cuáles nos informan de que el circuito cerebral no está fijado de forma unidireccional por la genética o las hormonas, sino que el sistema nervioso humano, tanto a nivel funcional como estructural, es un proceso dinámico y continuo que persiste a lo largo de la vida (Gergen, 2010; Ainsworth, 2015, McCarthy, 2016). En coherencia con esta plasticidad cerebral las miembros de NGN nos dicen en un artículo titulado precisamente "Plasticity, plasticity, plasticity. . . and the rigid problema of sex" (Fine et al, 2013, 551):

An understanding of gender as a complex, multilevel, hierarchical structure that shapes not only institutions, interrelations, cognition and perception, but also the brain, endocrine system, and the manifestation of evolutionary processes, can bring about better and more informative science ${ }^{8}$.

8 Traducción propia: "La comprensión del género como una estructura compleja, multinivel y jerárquica que moldea no sólo las instituciones, las interrelaciones, la cognición y la percepción, sino también el cerebro, el sistema endocrino y la manifestación de procesos evolutivos, puede producir una ciencia mejor y más informativa". 
La plasticidad del cerebro es de importancia crucial no sólo a nivel científico y epistemológico, sino a nivel político, ya que si lo social impacta en el cerebro es vital articular propuestas de cambio social y educativo que puedan conformar desarrollos cerebrales para un mundo más equitativo.

Otra cautela a tener en cuenta es que al informar de diferencias significativas entre los sexos, habrá de establecer y comunicar claramente el efecto de los tamaños de las muestras y los intervalos de confianza para asegurar tanto la fiabilidad de las conclusiones al respecto como la minimización de falsos positivos. Por ello se aconseja reducir el riesgo de falsos positivos que a veces son debidos a que los experimentos sólo toman cuenta de la categoría de sexo. Para prevenir este riesgo se aconseja trabajar con más variables siempre que sea posible. Y si esto no es posible el experimento en cuestión habría de mencionar y justificar por qué no lo es (Rippon et al, 2017, 2).

La pobreza estadística de algunos estudios ha sido la culpable, muchas veces, de reportar diferencias significativas cuando en realidad han sido "falsos positivos". Pensemos en el debate generado tras declaraciones de la conocida neuropsiquiatra Louann Brizendine (quien representa lo que algunos llaman "pop neurosciences"), quien en su bestseller The Female Brain (2006) concluyó que las mujeres pronuncian 20.000 palabras por día y los hombres "sólo" 7.000. El escándalo se precipitó al reconocer la autora que la muestra había sido de 10 sujetos: 5 niñas y 5 niños. Esta infra-representación fue corregida posteriormente por el estudio de Mehl et al (2007), al concluir, con un experimento con 396 participantes, que no hay diferencias por sexo en la cantidad de palabras que emitimos. Sin embargo, es notorio que las conclusiones acientíficas de Brizendine llenaron titulares en los medios de comunicación de masas, mientras que las del estudio de Mehl et al apenas tuvieron eco en tales medios.

En este sentido una de las peticiones constantes de gran parte de la comunidad científica que investiga el tema de las influencias sexuales en el cerebro es que es importante publicar no sólo las diferencias encontradas, sino también los fallos en la replicación de diferencias esperadas, así como de similitudes. Por ello ha habido una constante crítica entre la comunidad neurocientífica hacia la tendencia de las revistas científicas a publicar mayoritariamente informes sobre "diferencias sexuales", y no similitudes, entre los cerebros. Como contrapartida al proceso típico en gran parte acrítico (puesto que elige lo que se quiere contar en función de fuertes prejuicios, en este caso la diferencia cerebral de hombres y mujeres) las autoras recomiendan un proceso experimental alternativo que impida que los prejuicios puedan influir en el proceso científico.

Las autoras de la carta al editor de Journal of Neuroscience Research acentúan la importancia de discutir y reportar no sólo las diferencias, sino las similitudes (Rippon et al, 2017, $2)$, tal y como proponen Rippon et al $(2014,6)$ en el cuadro que mostramos como "proceso experimental recomendado". Nótese que en el proceso experimental típico sólo son considerados publicables los resultados que muestran diferencias, pues, como advierten las autoras, la investigación del cerebro, esencialmente a través de las técnicas de neuroimagen, han propulsado la tendencia a mantener creencias esencialistas acerca de las categorías sociales, principalmente las relacionadas con el sexo y el género (Rippon et al, 2014, 1). Esto puede verse claramente en el número de artículos publicados en las últimas dos décadas sobre el tema de las diferencias sexuales en el cerebro humano: se ha doblado el número en artículos científicos y se ha quintuplicado en el caso de artículos divulgativos (Maney, 2016). 
A. Proceso Experimental Típico

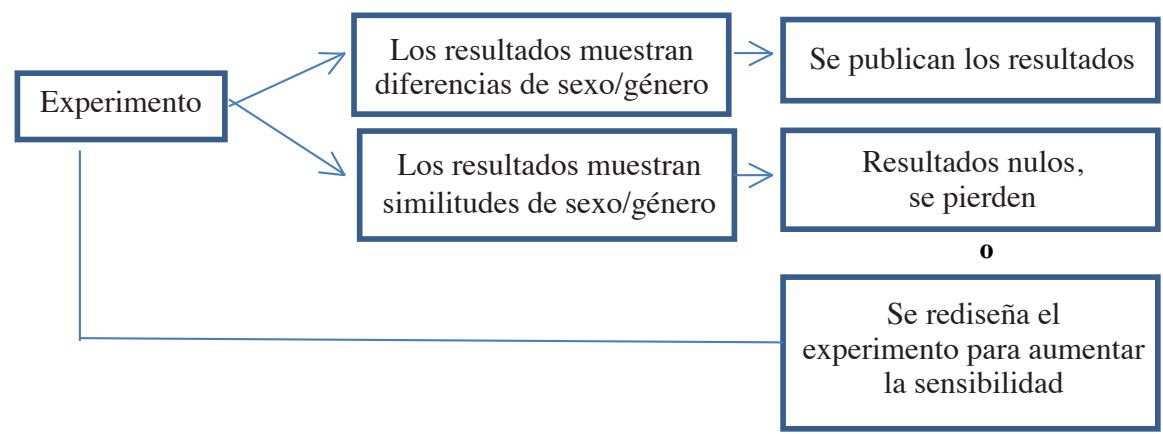

\section{B. Proceso Experimental Recomendado}

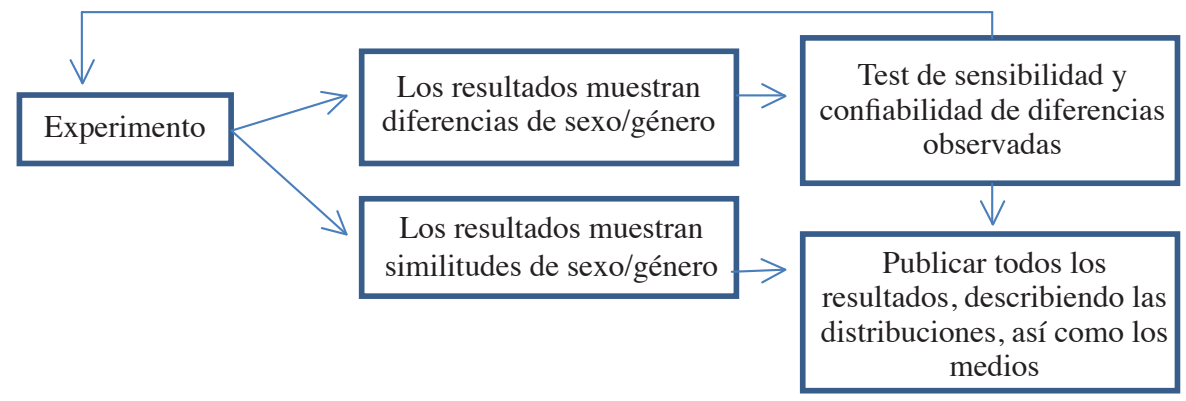

Cuadro propuesto por Rippon, Jordan-Young, Kaiser y Fine para la investigación en neuroimágenes $(2014,6)$ (Traducción propia).

Esto nos obliga a exigir que la comunicación, diseminación y divulgación de las conclusiones de los artículos científicos a la prensa y a los medios de comunicación en general sean realizadas por las revistas científicas con el rigor necesario. Las editoriales han de comprometerse con un mensaje apropiado y ajustado a las conclusiones científicas cuando elaboran sus comunicados de prensa o divulgan los hallazgos publicados en sus revistas. Como Maney observa $(2015,282)$ “..., because sex differences are so easily misunderstood and misinformation potentially harmful, we need to hold others and ourselves to a high standard when reporting them"9.

A estos problemas se ha de sumar la llamada "crisis de la reproducibilidad", que según un estudio publicado en Nature (Baker, 2016) supone que el 70\% de los científicos han fallado a la hora de reproducir experimentos de otros. La denuncia no es nueva: Simmons et al (2011) propusieron una guía que incluía la necesidad de replicación en algunos casos para evitar los falsos positivos. En esta misma línea el denominado “The Reproducibility

9 Traducción propia: “..., debido a que las diferencias sexuales son tan fácilmente malinterpretadas y la desinformación potencialmente dañina, necesitamos mantener a los demás ya nosotros mismos a un alto nivel al reportarlos". 
Project"10 (OSC, 2012) afirma un grave fallo de reproducibilidad en las ciencias psicológicas. El grupo que conforma este proyecto, 72 voluntarios de 41 instituciones, lleva adelante el proyecto de replicar de forma transparente estudios publicados desde 2008 en las tres principales revistas de psicología (muchos de esos estudios relacionados directa o indirectamente con las diferencias sexuales).

La monitorización al más alto nivel de los hallazgos científicos es una exigencia ineludible si pensamos las consecuencias que muchas conclusiones científicas tienen en nuestras vidas, personales y colectivas. Buena prueba de ello es el ejemplo con el que empezábamos este artículo en relación a las afirmaciones del Dr. Dana en 1915 sobre las mujeres y el derecho al voto. Actualmente, y en un contexto de creciente desigualdad en la distribución de recursos de todo tipo, afirmaciones falsamente científicas en relación a las diferencias de capacidades neuronales entre los sexos puede tener el efecto de sedimentación de discursos que reorganicen y renaturalicen la desigualdad de los géneros en base a las pretendidas diferencias cerebrales. Ciertamente no es lo mismo diferencia que desigualdad, y sabemos que las diferencias no han de conllevar desigualdades, como algunos neurocientíficos de la diferencia sexual también alertan en sus conclusiones (Cahill, 2017), pero no es menos cierto que hemos aprendido por experiencia que en el caso de la dominación de las mujeres toda diferencia etiquetada como "sexual" ha sido utilizada como fundamento para crear desigualdad. Será necesario, por ello, una profilaxis rigurosa a la hora de anunciar diferencias cerebrales entre los sexos, y a la hora de interpretar consecuencias comportamentales de esas diferencias.

\section{Conclusión}

La primera y más importante conclusión, a la vista de las recomendaciones de las revisiones y aclaraciones del NGN, es que es necesario descartar asunciones binarias en el mismo planteamiento de los experimentos. Ello imposibilita precisamente concluir si hay diferencias significativas a nivel cerebral entre los sexos, ya que parte de la hipótesis a la que se quiere llegar como conclusión.

Asimismo se hace necesario advertir de las cautelas que la comunicación de la ciencia ha de tomar en cuenta en el uso de la divulgación de los experimentos científicos (Maney, 2015). El uso de términos como "profundas", "fundamentales" o "esenciales" deberían ser descartados cuando se habla de pequeñas diferencias reportadas a través de experimentos que sólo trabajan con la variable de sexo, o que no cuentan con un tamaño representativo de muestra (Rippont et al, 2017). Este tipo de lenguaje es científicamente injustificado y resulta un grave inconveniente a la hora de poder avanzar la investigación neurocientífica (O'Connor y Joffe, 2014).

Por todo ello la propuesta metodológica que se infiere del trabajo de revisión y aclaración que el grupo de NeuroGenderings Network plantea nos parece pertinente y necesaria si el creciente campo de la investigación neurocientífica quiere aportar conclusiones sólidas para conformar un conocimiento, no sólo veraz y fiable, sino socialmente competente. La aceptación generalizada del discurso científico y su gran valoración por las sociedades,

10 Puede verse la planificación, coordinación y ejecución del proyecto en su página "Open Science Framework" (http://openscienceframework.org/). 
podríamos decir incluso su "seductor atractivo" (Skolnick Weisberg et al, 2008), vuelve a las neurociencias una especie de guía para mucha gente a la hora de entender sus propias vidas. Los excesos de las promesas "bio" y "neuro" relacionadas con la concepción omniabarcante de lo neuro-biológico han conllevado recientemente, según Rose y Abi-Rached (2013), una cierta apertura de las concepciones neurocientíficas, lo cual puede significar una nueva relación más productiva entre las neurociencias y las ciencias sociales. En este marco de diálogo crítico (Sprague, 2016) y de "neurociencias críticas" (Kraus, 2011) pensamos que las propuestas aquí planteadas desde la epistemología feminista pueden permitir algo que debe estar siempre presente en la investigación científica, el debate robusto y riguroso entre la comunidad científica. Ello permitirá la generación de mejor conocimiento, pero para ello sigue haciendo falta, como hemos pretendido mostrar, un "activismo científico" que se ocupe de una tarea de monitorización constante. Los vicios científicos y las asunciones prejuiciosas siguen estando hoy presentes en la actividad científica. Los grandes intereses económicos relacionados con la investigación científica junto a la deriva de las políticas científicas en cuanto a subvenciones pueden trasladarse a la comunidad científica en forma de presiones para favorecer un conocimiento científico que sea prometedor y de rápida aplicación. Estas presiones, englobadas en lo que comúnmente se denomina "sistema de incentivos científicos", afectan a la selección de cuestiones y temas de investigación, a la preparación y diseño de experimentos, a la interpretación de resultados y a la traducibilidad de esos resultados a la aplicación práctica. El peligro que conllevan estas presiones y estas malas prácticas es el de quebrantar el principio de una ciencia que sirva y proteja a las sociedades humanas y al mundo. En la denominada "era del cerebro" estas prácticas acientíficas pueden acarrear catastróficas consecuencias, como advierten diversas voces dentro de la misma comunidad científica (Littlefield y Johnson, 2012). Por ello, la tarea vigilante, a modo de "guerrilla epistemológica", es más que nunca necesaria.

\section{Referencias}

Ainsworth, Claire (2015): “Sex Redefined", Nature, 518, 19 February: 288-291.

Baker, Monya (2016): "1.500 scientists lift the lid on reproducibility. Survey sheds light on the 'crisis' rocking Research", Nature 533: 452-454 (26 May 2016).

Bleier, Ruth (1984): Science and Gender: A Critique of Biology and Its Theories on Women, Elsevier Science \& Technology Books, Amsterdam.

Brizendine, Louann (2006): The Female Brain, Broadway Books, Random House, New York.

Butler, Judith (1990): Gender Trouble, Routledge, New York.

Cahill, Larry (2017): “An issue whose time has come”, Journal of Neuroscience Research 95 (2017): 12-13.

Clayton, Janine A. \& Collins, Francis S. (2014): "NIH to balance sex in cell and animal studies", Nature 509 (7500): 282-283.

Davis, Lennard J. \& Morris, David B. (2007): "Biocultures Manifesto". New Literary History: a journal of theory and interpretation, 38(3): 411-418. 
Eliot, Lise \& Richardson, Sarah S. (2016): "Sex in Context: Limitations of Animal Studies for Addressing Human Sex/Gender Neurobehavioral Health Disparities", Journal of Neuroscience, 36 (47): 11823-11830.

Fausto-Sterling, Anne (2000): Sexing the Body: Gender Politics and the Construction of Sexuality, Basic Books, New York.

Fine, Cordelia (2008): "Will working mothers' brains explode? The popular new genre of neurosexism", Neuroethics 1 (1): 69-72.

Fine, Cordelia (2010): Delusions of Gender: How Our Minds, Society, and Neurosexism Create Difference, W. W. Norton, New York.

Fine, Cordelia \& Jordan-Young, Rebecca (2017): "We've been labelled 'anti-sex difference' for demanding greater scientific rigour", The Guardian, 6/4/2017.

Fine, Cordelia; Jordan-Young, Rebecca; Kaiser, Anelis \& Rippon, Gina (2013): "Plasticity, plasticity, plasticity and the rígid problema of sex", Trends in Cognitive Sciences, 17(11): 550-551.

Fox Keller, Evelyn (2010): The Mirage of a Space between Nature and Nurture, Duke University Press, Durkham \& London.

García Dauder, Silvia \& Pérez Sedeño, Eulalia (2017): Las 'mentiras' científicas sobre las mujeres, Los libros de la catarata, Madrid.

Gergen, Kenneth J. (2010): “The acculturated Brain", Theory \& Psychology 20 (6): 795-816.

Gottlieb, Gilbert (2000): "Environmental and behavioral influence on gene activity", Current Directions in Psychological Science, 9, 93-97.

Gottlieb, Gilbert (2002): "Developmental-behavioral initiation of evolutionary change", Psychological Review, 109: 211-218.

Greenfield, Susan (2000): The private life of the brain, Penguin Press, New York.

Grosz, Elizabeth (1994): Volatile Bodies: Toward a Corporeal Feminism, Indiana University Press, Bloomington.

Hansson, Sven Ove (2015): "Science and Pseudo-Science", The Stanford Encyclopedia of Philosophy (Spring 2015 Edition), Edward N. Zalta (ed.). Disponible: <http://plato. stanford.edu/archives/spr2015/entries/pseudo-science/>. Consultado 24 Febrero 2016.

Hubbard, Ruth (1990): The Politics of Women's Biology, New Brunswick, Rutgers University Press, New Jersey \& London.

Joel, Daphna et al (2015): "Sex beyond the genitalia: The human brain mosaic", Proceedings of the National Academy of Sciences (PNAS), 112 (50): 15468-15473.

Joel, Daphna \& Mccarthy, Margaret M. (2017): "Incorporating Sex As a Biological Variable in Neuropsychiatric Research: Where Are We Now and Where Should We Be?", Neuropsychopharmacology, (2017) 42: 379-385.

Kaiser, Anelis (2012): “Re-Conceptualizing 'Sex' and 'Gender' in the Human Brain”, Zeitschrift fur Psychologie, 220(2): 130-136.

Kraus, Cynthia (2011): "Critical Studies of the Sexed Brain: A Critique of What and for Whom?", Neuroethics, 5(3): 247-259.

Laqueur, Thomas (1994): La construcción del sexo. Cuerpo y genero desde los griegos hasta Freud, Cátedra, Colección Feminismos, Madrid. Traducción del original de 1990 por Eugenio Portela. 
Li, Schu-Chen (2003): "Biocultural Orchestration of Developmental Plasticity Across Levels: The Interplay of Biology and Culture in Shaping the Mind and Behavior Across the Life Span", Psychological Bulletin of the American Psychological Association, 129(2): 171-194.

Littlefield, Melissa \& Johnson, Jenell, eds. (2012): Neuroscientific Turn: Transdisciplinarity in the Age of the Brain, The University Of Michigan Press, Ann Arbor.

Maney, Donna L. (2015): "Just like a circus: the public consumption of sex differences", Current Topics in Behavioral Neurosciences, 19: 279-96.

Maney, Donna L. (2016): "Perils and pitfalls of reporting sex differences", Philosophical Transactions B. The Royal Society Publishing, 371(1688): 20150119.

Mccarthy, Margaret M. (2016): "Multifaceted origins of sex differences in the brain", Philosophical Transactions B. The Royal Society Publishing. 371(1688): 20150106.

Mehl, Matthias R.; Vazire, Simine; Ramírez-Esparza, Nairán; Slatcher, Richard B.; Pennebaker, James W. (2007): “Are Women Really More Talkative Than Men?”, Science, 6 Jul 2007: 82 .

Nicholson, Linda (1994): “Interpreting Gender”, Signs. Journal of Women in Culture and Society, 20(1): 79-105.

O'Connor, Cliodhna \& Joffe, Helene (2014): “Gender on the Brain: A Case Study of Science Communication in the New Media Environment", PLoS ONE, 9(10): e110830.

OSC Open Science Collaboration (2012): "Estimating the Reproducibility of Psychological Science", Reproducibility Project: Psychology, Open Science Framework. Disponible en $<$ https://osf.io/ezcuj/> Consultado el 21 diciembre 2016.

Prager, Eric M. (2017): “Addressing Sex as a Biological Variable”, Journal of Neuroscience Research, 95 (2017): 11.

Reverter-Bañón, Sonia (2016): "Reflexión crítica frente al Neurosexismo", Pensamiento, 72(273): 959-979.

Ridley, Matt (2003): Nature via nurture: Genes, experience, and what makes us human, HarperCollins Publishers, New York.

Rippon, Gina, Jordan-Young, Rebecca, Kaiser, Anelis \& Fine, Cordelia (2014): "Recommendations for sex/gender neuroimaging research: key principles and implications for research design, analysis, and interpretation", Frontiers in Human Neurosciences, 8: 650.

Rippon, Gina, Jordan-Young, Rebbecca, Kaiser, Anelis, Joel, Daphna \& Fine, Cordelia (2017): "Journal of neuroscience research policy on addressing sex as a biological variable: Comments, clarifications, and elaborations", Journal of Neuroscience Research. Doi:10.1002/jnr.24045 (solo online). Consultado 4-3-2017.

Rose, Nikolas \& Abi-Rached, Joelle M. (2013): Neuro: The New Brain Sciences and the Management of the Mind, Princeton University Press, Princeton NJ.

Russett, Cynthia E (1989): Sexual Science: The Victorian Construction of Womanhood, Harvard University Press, Cambridge MA.

Schmitz, Sigrid (2012): "The neurotechnological cerebral subject: Persistence of implicit and explicit gender norms in a network of change", Neuroethics, 5(3): 261-274.

Scott, Anne (1997): “The Knowledge in Our Bones: Standpoint Theory, Alternative Health and the Quantum Model of the Body", Maynard, Mary, ed., Science and the Construction of Women, University College London, London, pp. 106-125. 
Severin, Laura \& Wyer, Mary (2000): “The Science and Politics of the Search for Sex Differences: editorial", NWSA Journal, 12(3): vii-xvi.

Simmons, Joseph P., Nelson, Leif D., Simonsohn, Uri (2011): "False-Positive Psychology. Undisclosed Flexibility in Data Collection and Analysis Allows Presenting Anything as Significant", Psychological Science, 22(11): 1359-1366.

Skolnick Weisberg, D., Keil, F.C., Goodstein, J., Rawson, E., \& Gray, J. (2008): “The seductive allure of neuroscience explanations", Journal of Cognitive Neuroscience, 20: 470-477.

Spelman, Elizabeth V. (2007): "Managing Ignorance", en Sullivan, Shanon \& Tuana, Nancy, eds. Race and Epistemologies of Ignorance, Suny Press, New York, pp. 119-134.

Spivak, Gayatri Ch. (1993): Outside in the Teaching Machine, Routledge, New York \& London.

Sprague, Joey (2016, 2005): Feminist Methodologies for Critical Researchers: Bridging Differences, Rowman \& Littlefield, London.

Tobach, Ethel \& Rosoff, Betty, eds. (1994): Challenging racism \& sexism: Alternatives to genetic explanations, The Feminist Press, Cuny Press, New York.

Tuana, Nancy (2004): "Coming to understand: Orgasm and the epistemology of ignorance, Hypatia: A Journal of Feminist Philosophy, 19(1): 194-232.

Tuana, Nancy (2006): “The Speculum of Ignorance: The Women's Health Movement and Epistemologies of Ignorance", Hypatia: A Journal of Feminist Philosophy, 21(3): 1-19. Wood, Jane (2001): Passion and Pathology in Victorian Fiction, Oxford University Press. 